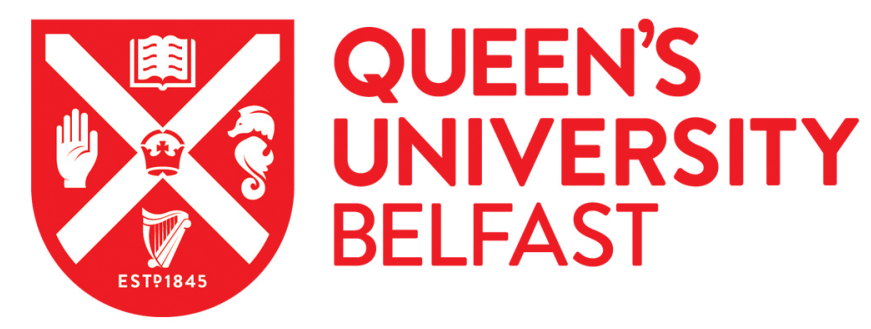

\title{
Orthogonal Vector Approach for Synthesis of Multi-beam Directional Modulation Transmitters
}

Ding, Y., \& Fusco, V. (2015). Orthogonal Vector Approach for Synthesis of Multi-beam Directional Modulation Transmitters. IEEE Antennas and Wireless Propagation Letters, 14, 1330-1333.

https://doi.org/10.1109/LAWP.2015.2404818

\section{Published in:}

IEEE Antennas and Wireless Propagation Letters

\section{Document Version:}

Peer reviewed version

\section{Queen's University Belfast - Research Portal:}

Link to publication record in Queen's University Belfast Research Portal

\section{Publisher rights}

( 2015 IEEE. Personal use of this material is permitted. Permission from IEEE must be obtained for all other users, including reprinting/ republishing this material for advertising or promotional purposes, creating new collective works for resale or redistribution to servers or lists, or reuse of any copyrighted components of this work in other works.

\section{General rights}

Copyright for the publications made accessible via the Queen's University Belfast Research Portal is retained by the author(s) and / or other copyright owners and it is a condition of accessing these publications that users recognise and abide by the legal requirements associated with these rights.

Take down policy

The Research Portal is Queen's institutional repository that provides access to Queen's research output. Every effort has been made to ensure that content in the Research Portal does not infringe any person's rights, or applicable UK laws. If you discover content in the Research Portal that you believe breaches copyright or violates any law, please contact openaccess@qub.ac.uk. 


\title{
Orthogonal Vector Approach for Synthesis of Multi-beam Directional Modulation Transmitters
}

\author{
Yuan Ding and Vincent Fusco, Fellow, IEEE
}

\begin{abstract}
An orthogonal vector approach is proposed for the synthesis of multi-beam directional modulation (DM) transmitters. These systems have the capability of concurrently projecting independent data streams into different specified spatial directions while simultaneously distorting signal constellations in all other directions. Simulated bit error rate (BER) spatial distributions are presented for various multi-beam system configurations in order to illustrate representative examples of physical layer security performance enhancement that can be achieved.
\end{abstract}

Index Terms - Bit error rate (BER), directional modulation (DM), orthogonality, security.

\section{INTRODUCTION}

$\mathrm{D}$ rectional modulation $(\mathrm{DM})$ is a transmitter side technology that is capable of projecting digitally encoded information signals into a pre-specified spatial direction while simultaneously distorting the constellation formats of the same signals in all other directions in free space [1]. This emerging technology, as a promising keyless physical-layer security means, has developed in many aspects in recent years [1] [20]. Specifically in [1] a vector model for DM transmitters was described, from which rigorous necessary conditions for achieving DM characteristics were derived. From this point of view DM transmitters obeying the necessary conditions (5) and (6) in [1] cannot be synthesized with the passive DM architectures [2], [3], termed by authors near-field direct antenna modulation (NFDAM), due to the complex transformation between the near-field electromagnetic boundaries and far-field radiation characteristics. In order to solve this problem the actively excited DM arrays [4], [5] and the corrected bit-error-rate-driven (BER-driven) synthesis approaches [6] were introduced. More studies on the performance of this type of DM arrangement were conducted in [7]-[10]. Another important aspect, i.e., performance assessment metrics for DM systems, was formally investigated in [11], wherein BER obtained by transmitting a certain length of random data symbols was considered as an appropriate metrics for both static and dynamic DM systems performance evaluation. Besides the BER-driven synthesis method presented in [6], several other DM synthesis approaches based on the actively excited antenna arrays were developed, e.g., the orthogonal vector approach in [1], the constrained far-field radiation pattern approaches in [12], [13], and the far-field

Manuscript received January 04, 2015. This work was sponsored by the Queen's University of Belfast High Frequency Research Scholarship.

Yuan Ding and Vincent Fusco are with the Institute of Electronics, Communications and Information Technology (ECIT), Queen's University of Belfast, Belfast, United Kingdom, BT3 9DT (phone: +44(0)2890971806; fax: +44(0)2890971702; e-mail: yding03@qub.ac.uk; v.fusco@qub.ac.uk). radiation pattern separation approach in [14]. All these methods are compatible to the digital DM architecture illustrated in [1]. Some other DM physical arrangements employing radio frequency (RF) switch arrays or Fourier beam-forming lens were studied in [15]-[18]. These antenna array feeding networks, from the orthogonal vector's point of view, can be regarded as hardware implementations to achieve orthogonality between information and interference.

It is noted that all the above mentioned DM work is restricted to single-beam DM systems where only one information data stream can be securely conveyed along one prescribed direction in free space. It is natural to consider developing multi-beam DM systems which have the capability of concurrently projecting multiple independent information data streams into different spatial directions, while simultaneously distorting information signal formats along all other unselected directions.

The preliminary multi-beam DM synthesis attempts based on the DM arrays with 2-bit phase shifters or the far-field pattern separation approach can be found in [19] and [20]. In order to generalize the multi-beam DM transmitter synthesis, in Section II an orthogonal vector concept is developed for multi-beam DM synthesis. A synthesis example is provided in Section III. System bit error rate (BER) simulations are presented in Section IV. Finally conclusions are drawn in Section V.

\section{ORthogonal Vector Multi-BEAm DM SYNTHESIS}

In this section, a one-dimensional (1-D) $N$-element half wavelength spaced antenna array with each element having an ideal isotropic radiation pattern is used as the discussion example. The array phase center is located at the array's geometric center. The synthesis of a dual-beam DM transmitter is described. The procedure developed can be readily extended to $N-1$ beam DM synthesis. It should be noted that the dual-beam DM system presented in this paper is completely un-related to the 'dual-beam' DM architecture in [21], which utilizes two beams transmitted along the same direction to construct a single-beam DM transmitter.

We assume that two independent data streams are to be conveyed along different spatial directions $\alpha$ and $\beta\left(\alpha, \beta \in\left[0^{\circ}\right.\right.$, $\left.\left.180^{\circ}\right] ; \alpha \neq \beta\right)$. The channel vectors along these directions are

$$
\begin{aligned}
& \boldsymbol{H}_{o v}(\alpha)=[\underbrace{e^{j \frac{N-1}{2} \pi \cos \alpha}}_{H_{\text {ov } \_\alpha}} \underbrace{e^{j\left(\frac{N-1}{2}-1\right) \pi \cos \alpha}}_{H_{\text {ov } \_\alpha 2}} \cdots \underbrace{e^{j\left(\frac{N-1}{2}-(N-1)\right) \pi \cos \alpha}}_{H_{\text {ov } \_\alpha N}}]^{T} \\
& \boldsymbol{H}_{o v}(\beta)=[\underbrace{e^{j \frac{N-1}{2} \pi \cos \beta}}_{H_{o v_{-} \beta 1}} \underbrace{e^{j\left(\frac{N-1}{2}-1\right) \pi \cos \beta}}_{H_{o v_{-} \beta 2}} \cdots \underbrace{e^{j\left(\frac{N-1}{2}-(N-1)\right) \pi \cos \beta}}_{H_{o v_{-} \beta N}}]^{T}
\end{aligned}
$$


Operator ' $[\cdot]^{T}$, denotes vector transpose. Combining $\boldsymbol{H}_{o v}(\alpha)$ and $\boldsymbol{H}_{o v}(\beta)$ as a matrix $\left[\boldsymbol{H}_{o v}\right]$ gives,

$$
\left[\boldsymbol{H}_{o v}\right]=\left[\begin{array}{llll}
H_{o v_{-} \alpha 1} & H_{o v_{-} \alpha 2} & \cdots & H_{o v_{-} \alpha N} \\
H_{o v_{-} \beta 1} & H_{o v_{-} \beta 2} & \cdots & H_{o v_{-} \beta N}
\end{array}\right]^{T}
$$

The array excitation vectors, $\boldsymbol{\Lambda}_{\alpha}$ and $\boldsymbol{\Lambda}_{\beta}$, for information data transmitted along $\alpha$ and $\beta$ respectively are obtained using (4) and (5). These two formulas are associated with the pattern projection method [22], [23], by which the far-field radiation pattern excited by $\boldsymbol{\Lambda}_{\alpha}$ (or $\boldsymbol{\Lambda}_{\beta}$ ) has a null along direction $\beta$ (or $\alpha)$. In other words, two corresponding excited far-field patterns are orthogonal to each other along their prescribed directions, meaning that two information data streams, when radiated through these two far-field patterns respectively, can be conveyed along their selected directions without crossover. In (4) and (5) $\boldsymbol{I}_{N}$ denotes the $N \times N$ identity matrix, and the ' $[\cdot]^{\dagger}$ ', denotes the complex conjugate transpose (Hermitian) operator. The ' $[\cdot]^{-1}$, is the Moore-Penrose pseudo-inverse operator, returning an $N \times 1$ vector, while $1 /\left\|\boldsymbol{H}_{o v}(\alpha)\right\|^{2}$ and $1 /\left\|\boldsymbol{H}_{o v}(\beta)\right\|^{2}$ provide power normalization. Operator ' $\|\cdot\|$ ' returns the norm of the enclosed vector.

$$
\begin{aligned}
\Lambda_{\alpha} & =\left[\begin{array}{llll}
\Lambda_{\alpha 1} & \Lambda_{\alpha 2} & \cdots & \Lambda_{\alpha N}
\end{array}\right]^{T} \\
& =\frac{1}{\left\|\boldsymbol{H}_{o v}(\alpha)\right\|^{2}}\left[\boldsymbol{I}_{N}-\left(\boldsymbol{H}_{o v}^{\dagger}(\beta)\right)^{-1} \boldsymbol{H}_{o v}^{\dagger}(\beta)\right] \boldsymbol{H}_{o v}(\alpha) \\
\boldsymbol{\Lambda}_{\beta} & =\left[\begin{array}{llll}
\Lambda_{\beta 1} & \Lambda_{\beta 2} & \cdots & \Lambda_{\beta N}
\end{array}\right]^{T} \\
& =\frac{1}{\left\|\boldsymbol{H}_{o v}(\beta)\right\|^{2}}\left[\boldsymbol{I}_{N}-\left(\boldsymbol{H}_{o v}^{\dagger}(\alpha)\right)^{-1} \boldsymbol{H}_{o v}^{\dagger}(\alpha)\right] \boldsymbol{H}_{o v}(\beta)
\end{aligned}
$$

Finally the dual-beam DM transmitter array excitations $\boldsymbol{S}_{i}$ can be obtained using (6),

$$
\boldsymbol{S}_{i}=B_{i} \boldsymbol{\Lambda}_{\alpha}+Q_{i} \boldsymbol{\Lambda}_{\beta}+\boldsymbol{W}_{o v}
$$

Here $B_{i}$ and $Q_{i}$ are the $i^{\text {th }}$ symbol in each of the two independent information data streams along directions $\alpha$ and $\beta$, respectively. Each of them can be modulated with any types of digital modulation. For simplicity in what follows we assume that both data streams have the same symbol rate. $\boldsymbol{W}_{o v}$ is the orthogonal vector, which is updated in the null space of the $\left[\boldsymbol{H}_{o v}^{*}\right]$ at the information data rate, i.e., a dynamic DM system. Hence $\boldsymbol{W}_{o v}$ is simultaneously orthogonal to both $\boldsymbol{H}_{o v}^{*}(\alpha) \quad$ and $\quad \boldsymbol{H}_{o v}^{*}(\beta), \quad$ i.e., $\quad \boldsymbol{H}_{o v}^{*}(\alpha) \cdot \boldsymbol{W}_{o v}=0 \quad$ and $\boldsymbol{H}_{o v}^{*}(\beta) \cdot \boldsymbol{W}_{o v}=0$. In other words, the injected orthogonal vector $\boldsymbol{W}_{o v}$ has no effect on the information signals projected along the pre-specified spatial directions $\alpha$ and $\beta$. The ' $[\cdot]^{* \text { ', is }}$ the complex conjugate operator.

It should be noted that the pattern separation synthesis approach described in [20] is closely linked to the universal orthogonal vector approach proposed in this section. In fact the information patterns used in [20] can be considered as patterns associated with excitations $\boldsymbol{\Lambda}_{\alpha}$ or $\boldsymbol{\Lambda}_{\beta}$, while the interference patterns defined in [20] can be regarded as patterns excited by certain particular orthogonal vectors $\boldsymbol{W}_{o v}$. However, these two methods are different in the following key aspects;

- DM transmitters synthesized by the pattern separation approach focus interference power along several prespecified directions, while the orthogonal vector approach spreads the interference power across the whole spatial space other than the secure communication directions.

- The orthogonal vector approach has the ability to precisely control the DM power efficiency, $\mathrm{PE}_{\mathrm{DM}}$, which describes what percentage of total radiated energy is utilized for useful information transmission, definition as (25) in [1], while the pattern separation approach cannot.

- The orthogonal vector approach can be readily extended for multipath applications by replacing free space channel vectors with the multipath channel vectors, while the pattern separation approach only works for the free space scenario.

\section{SYNTHESIS EXAMPLE}

In the following synthesis example, it is assumed that a signal stream modulated with BPSK $\left(B_{i}\right)$ are projected along $\alpha$, $45^{\circ}$, while another independent signal stream $\left(Q_{i}\right)$ with the same power and symbol rate, modulated with Gray-coded QPSK, is transmitted along $\beta, 90^{\circ}$ (boresight), by a 5-element $(N=5)$ antenna array.

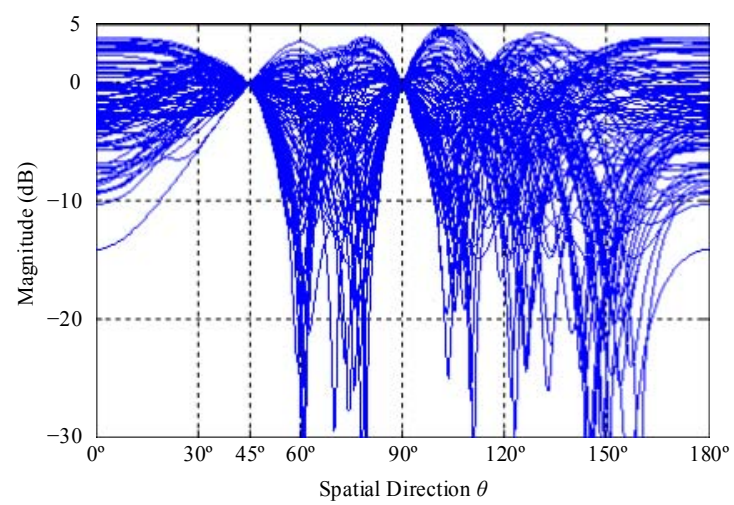

(a)

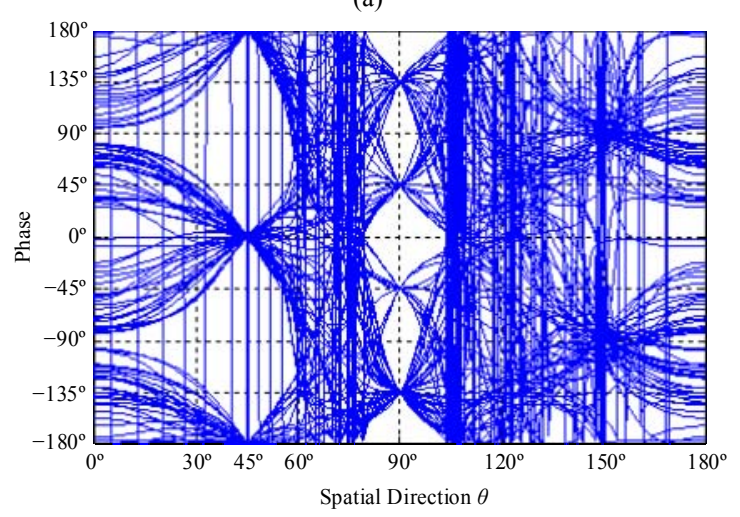

(b)

Fig. 1. Far-field radiation (a) magnitude and (b) phase patterns for 100 random transmission states in the synthesized dual-beam BPSK-QPSK dynamic DM system. $\mathrm{PE}_{\mathrm{DM}}=33 \%$. 
When the injected orthogonal vector $\boldsymbol{W}_{o v}$, which is updated in the null space of $\left[\boldsymbol{H}_{o v}^{*}\right]$, in (6) varies for each unique transmission state $(2 \times 4=8$ unique states in a dual-beam BPSK-QPSK DM system), a dual-beam dynamic DM transmitter will have been synthesized. Fig. 1 illustrates the far-field radiation patterns for 100 random transmission states, which are calculated by $\boldsymbol{G}^{\dagger} \boldsymbol{S}_{i}$. $\boldsymbol{G}$ is expressed similarly as $\boldsymbol{H}_{o v}(\alpha)$ in (1) with $\alpha$ replaced by $\theta . \theta \in\left[0^{\circ}, 180^{\circ}\right]$. The $\mathrm{PE}_{\mathrm{DM}}$ is set to $33 \%$. Since one signal stream, e.g., $B_{i}$, acts as the orthogonal interference to the other one, e.g., $Q_{i}$, and the signal strength of each is set to be identical, the $P E_{D M}$ value of $33 \%$ indicates that the additional injected orthogonal vector $\boldsymbol{W}_{o v}$ has the same power as each of the transmitted information signals. It is noted that phases are wrapped along direction $\alpha$, $45^{\circ}$, when the BPSK symbol ' 0 ' is sent, Fig. 1 (b).

The far-field radiation patterns when viewed from the receiver side can be translated to constellation symbols in inphase and quadrature (IQ) space when detected by receivers located along each spatial direction. Thus from Fig. 1 it can be observed that standard BPSK and QPSK constellation patterns are formed only along the prescribed spatial directions, $45^{\circ}$ and $90^{\circ}$ as required, with the signal IQ formats along all other directions being distorted, in such a fashion to lower the possibility of unwanted interception by eavesdroppers located in these regions.

One possible array excitation set for transmission of each of the 8 unique states in the dual-beam BPSK-QPSK DM system is extracted from Fig. 1 and is listed in Table I. These excitations, for the interested readers, can be used to construct a static dual-beam BPSK-QPSK DM transmitter, again PE DM is set to $33 \%$.

TABLE I

SAMPLE DUAL-BEAM 1-D 5-ELEMENT STATIC DM ARRAY EXCITATIONS $\boldsymbol{S}_{i}{ }^{\text {a }}$ FOR SIMULTANEOUSLY BPSK AND GRAY-CODED QPSK TRANSMISSIONS ALONG SPATIAL DIRECTIONS OF $45^{\circ}$ AND $90^{\circ}$ RESPECTIVELY.

\begin{tabular}{|c|c|c|c|c|c|c|}
\hline & & $\begin{array}{c}S_{i 1} \\
\left(\times 10^{-1}\right)\end{array}$ & $\begin{array}{c}S_{i 2} \\
\left(\times 10^{-1}\right)\end{array}$ & $\begin{array}{c}S_{i 3} \\
\left(\times 10^{-1}\right)\end{array}$ & $\begin{array}{c}S_{i 4} \\
\left(\times 10^{-1}\right)\end{array}$ & $\begin{array}{c}S_{i 5} \\
\left(\times 10^{-1}\right)\end{array}$ \\
\hline $\mathbb{I}$ & $\begin{array}{l}\text { BPSK ' } 1 \text { ' } \\
\text { QPSK ' } 11 \text { ' }\end{array}$ & $\begin{array}{c}2.317 \\
+j 2.459\end{array}$ & $\begin{array}{c}0.850 \\
+j 1.413\end{array}$ & $\begin{array}{c}3.004 \\
+j 1.999\end{array}$ & $\begin{array}{c}-0.402 \\
-j 4.664\end{array}$ & $\begin{array}{c}2.145 \\
+j 5.708\end{array}$ \\
\hline $\mathbb{N}_{\sim}$ & $\begin{array}{l}\text { BPSK ' } 1 \text { ' } \\
\text { QPSK ' } 01 \text { ' }\end{array}$ & $\begin{array}{c}0.168 \\
+j 0.236\end{array}$ & $\begin{array}{r}-3.277 \\
+j 7.381\end{array}$ & $\begin{array}{c}0.129 \\
-j 0.116\end{array}$ & $\begin{array}{c}-1.301 \\
-j 0.648\end{array}$ & $\begin{array}{l}-2.634 \\
+j 0.062\end{array}$ \\
\hline$\pi_{\omega}$ & $\begin{array}{l}\text { BPSK ' } 1 \text { ' } \\
\text { QPSK '00' }\end{array}$ & $\begin{array}{c}0.307 \\
-j 5.245\end{array}$ & $\begin{array}{r}-3.309 \\
+j 0.002\end{array}$ & $\begin{array}{c}0.010 \\
-j 4.692\end{array}$ & $\begin{array}{l}-1.306 \\
+j 0.915\end{array}$ & $\begin{array}{r}-2.616 \\
+j 2.105\end{array}$ \\
\hline$\pi$ & $\begin{array}{l}\text { BPSK ' } 1 \text { ' } \\
\text { QPSK ' } 10 \text { ' }\end{array}$ & $\begin{array}{c}0.510 \\
-j 6.370\end{array}$ & $\begin{array}{c}0.097 \\
+j 2.716\end{array}$ & $\begin{array}{c}5.049 \\
-j 1.370\end{array}$ & $\begin{array}{c}1.014 \\
+j 0.497\end{array}$ & $\begin{array}{c}0.245 \\
-j 2.388\end{array}$ \\
\hline $\mathbb{u}^{i}$ & $\begin{array}{l}\text { BPSK ' } 0 \text { ' } \\
\text { QPSK ' } 11 \text { ' }\end{array}$ & $\begin{array}{c}3.355 \\
+j 3.686\end{array}$ & $\begin{array}{c}1.124 \\
+j 4.534\end{array}$ & $\begin{array}{l}-1.217 \\
-j 0.116\end{array}$ & $\begin{array}{c}3.101 \\
+j 2.200\end{array}$ & $\begin{array}{c}0.552 \\
-j 3.388\end{array}$ \\
\hline ii & $\begin{array}{l}\text { BPSK ' } 0 \text { ' } \\
\text { QPSK ' } 01 \text { ' }\end{array}$ & $\begin{array}{c}-1.331 \\
+j 0.608\end{array}$ & $\begin{array}{r}-0.983 \\
+j 1.772\end{array}$ & $\begin{array}{r}-2.796 \\
+j 1.879\end{array}$ & $\begin{array}{c}0.603 \\
+j 6.588\end{array}$ & $\begin{array}{l}-2.407 \\
-j 3.933\end{array}$ \\
\hline iì & $\begin{array}{l}\text { BPSK ‘ } 0 \text { ’ } \\
\text { QPSK ‘ } 00 \text { ' }\end{array}$ & $\begin{array}{l}-2.850 \\
+j 3.610\end{array}$ & $\begin{array}{c}0.983 \\
-j 3.764\end{array}$ & $\begin{array}{l}-3.822 \\
+j 0.733\end{array}$ & $\begin{array}{l}-1.697 \\
-j 3.794\end{array}$ & $\begin{array}{c}0.472 \\
-j 3.699\end{array}$ \\
\hline$\pi$ & $\begin{array}{l}\text { BPSK ‘0’ } \\
\text { QPSK ‘ } 10 \text { ’ }\end{array}$ & $\begin{array}{c}0.343 \\
+j 2.941\end{array}$ & $\begin{array}{c}3.433 \\
-j 5.340\end{array}$ & $\begin{array}{l}-0.961 \\
-j 0.022\end{array}$ & $\begin{array}{c}0.875 \\
-j 3.499\end{array}$ & $\begin{array}{c}3.225 \\
-j 0.994\end{array}$ \\
\hline
\end{tabular}

\section{BER SimULATION RESULtS}

In order to further evaluate the performance of the synthesized dual-beam DM system example in Sections III its BER properties are simulated by transmitting two parallel data streams each consisting of $10^{7}$ random symbols. For each case the AWGN contribution is identical in all spatial directions. In the BER simulations it is assumed that legitimate receivers and eavesdroppers are located at a same distance away from transmitters, which for free space leads to an identical path loss associated with them and makes calculated array factors usable as signal power spatial distributions. The details of the BER calculation method can be found in [11]. One difference to the single-beam DM case is that receiver types need to be clearly specified when illustrating BER spatial distribution results.

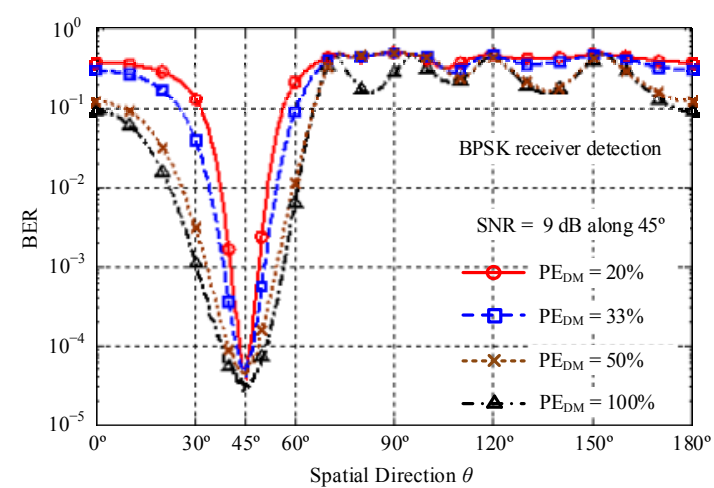

(a)

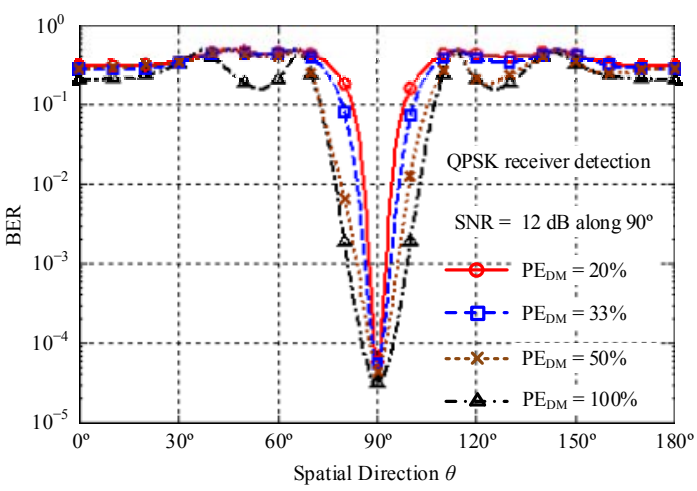

(b)

Fig. 2. BER simulation results detected by standard (a) BPSK and (b) QPSK receivers in the dual-beam BPSK-QPSK DM system. SNRs for BPSK and QPSK receiver cases are set to $9 \mathrm{~dB}$ and $12 \mathrm{~dB}$, respectively.

In Fig. 2 and Fig. 3 the BER simulation results obtained for standard BPSK and QPSK receiver detections in the dualbeam BPSK-QPSK DM systems are depicted. When the system noise is fixed the different signal to noise ratios (SNRs) can also be interpreted as different distance between the transmitter and receivers, including both legitimate nodes and eavesdropper nodes. For example a $10 \mathrm{~dB}$ SNR increase corresponds to a decrease of distance by a factor of $\sqrt{10}$. For comparison the BER responses of the corresponding conventional single-beam beam-steering system are also provided, which are equivalent to the case of $\mathrm{PE}_{\mathrm{DM}}=100 \%$. $\mathrm{PE}_{\mathrm{DM}}=50 \%$ refers to the dual-beam DM system with no additional orthogonal interference $\boldsymbol{W}_{o v}$ injected, i.e., only two streams of independent information signals of the same power are transmitted. The orthogonal interference in the dual-beam DM systems with $\mathrm{PE}_{\mathrm{DM}} \mathrm{S}$ of $20 \%$ and $33 \%$ is kept at constant 
power levels but is randomly updated at the symbol rate. The SNRs for the BPSK receiver detection are set $3 \mathrm{~dB}$ lower than those for the QPSK receiver cases in order to achieve similar BER value ranges along their pre-specified communication directions.

From Fig. 2 and Fig. 3 it can be concluded that the 'interference' contributed by one stream of information signals alone, $\mathrm{PE}_{\mathrm{DM}}=50 \%$, yields limited improvement on the secrecy performance for the transmission of the other information signal stream. This is because there are only a few states for the 'interference' to select. The number of states equals the number of the unique symbols in the 'interference' signal, e.g., 2 for BPSK and 4 for QPSK. Whereas inclusion of additional random orthogonal interference $\boldsymbol{W}_{o v}$ has the ability to narrow the main BER beams as well as suppress BER sidelobes, especially under high SNR scenarios. The more interference injected, the lower the possibility of data recovery from the leaked information signals radiated along unselected directions.

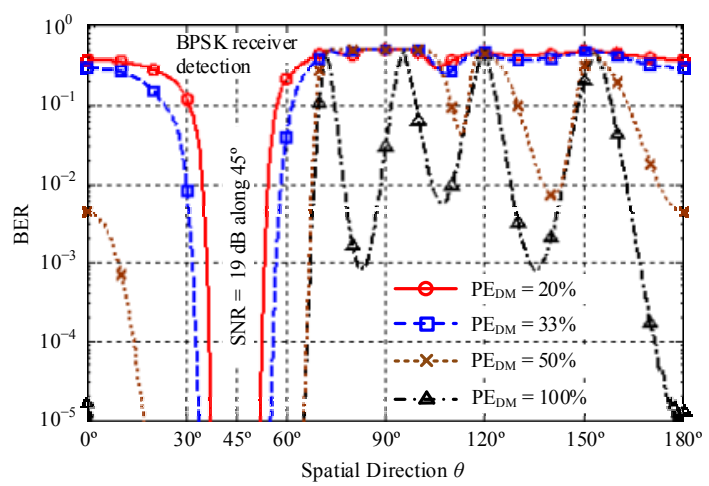

(a)

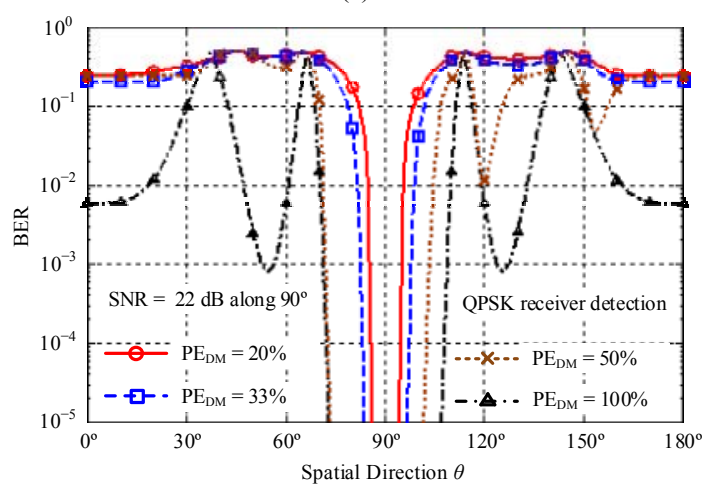

(b)

Fig. 3. BER simulation results detected by standard (a) BPSK and (b) QPSK receivers in the dual-beam BPSK-QPSK DM system. SNRs for BPSK and QPSK receiver cases are set to $19 \mathrm{~dB}$ and $22 \mathrm{~dB}$, respectively.

\section{CONCLUSION}

In this paper the orthogonal vector concept has been used for the synthesis of multi-beam DM systems. A dual-beam DM example with BER simulations validates the proposed synthesis method. The approach given here extends the use of DM transmitters from previous point to point only operation, to now include point to multipoint physical layer secured wireless transmission scenarios.

\section{REFERENCES}

[1] Y. Ding and V. Fusco, "A vector approach for the analysis and synthesis of directional modulation transmitters," IEEE Trans. Antennas Propag., vol. 62 , no. 1, pp. 361-370, Jan. 2014.

[2] A. Babakhani, D. B. Rutledge, and A. Hajimiri, "Transmitter architectures based on near-field direct antenna modulation," IEEE $J$. Solid-State Circuits, vol. 43, no. 12, pp. 2674-2692, Dec. 2008.

[3] A. Babakhani, D. Rutledge, and A. Hajimiri, "Near-field direct antenna modulation," IEEE Microw. Mag., vol. 10, no. 1, pp. 36-46, Feb. 2009.

[4] M. P. Daly and J. T. Bernhard, "Directional modulation technique for phased arrays," IEEE Trans. Antennas Propag., vol. 57, no. 9, pp. 26332640, Sep. 2009.

[5] M. P. Daly and J. T. Bernhard, "Beamsteering in pattern reconfigurable arrays using directional modulation," IEEE Trans. Antennas Propag., vol. 58, no. 7, pp. 2259-2265, Jul. 2010.

[6] Y. Ding and V. Fusco, "BER driven synthesis for directional modulation secured wireless communication," Int. J. Microw. Wireless Technol., vol. 6, no. 2, pp. 139-149, Apr. 2014.

[7] H. Z. Shi and T. Alan, "Direction dependent antenna modulation using a two element array," in Proc. 5th Eur. Conf. Antennas Propag. (EUCAP), 2011, pp. 812-815.

[8] H. Z. Shi and A. Tennant, "An experimental two element array configured for directional antenna modulation," in Proc. 6th Eur. Conf. Antennas Propag. (EUCAP), 2012, pp. 1624-1626.

[9] H. Shi and A. Tennant, "Enhancing the security of communication via directly modulated antenna arrays," IET Microw., Antennas Propag., vol. 7, no. 8, pp. 606-611, Jun. 2013.

[10] H. Shi and A. Tennant, "Covert communication using a directly modulated array transmitter," in Proc. 8th Eur. Conf. on Antennas and Propagation (EUCAP), 2014, pp. 316-318, 2014.

[11] Y. Ding and V. Fusco, "Establishing metrics for assessing the performance of directional modulation systems," IEEE Trans. Antennas Propag., vol. 62, no. 5, pp. 2745-2755, 2014.

[12] Y. Ding and V. Fusco, "Directional modulation transmitter radiation pattern considerations," IET Microw., Antennas Propag., vol. 7, no. 15, pp. 1201-1206, Dec. 2013.

[13] Y. Ding and V. Fusco, "Constraining directional modulation transmitter radiation patterns," IET Microw., Antennas Propag., vol. 5, no. 18, pp. 1408-1415, Dec. 2014.

[14] Y. Ding and V. Fusco, "A far-field pattern separation approach for the synthesis of directional modulation transmitter arrays," in Proc. XXXIth URSI General Assembly and Scientific Symposium (GASS), 2014, doi: 10.1109/URSIGASS.2014.6929295

[15] T. Hong, M. Z. Song, and Y. Liu, "RF directional modulation technique using a switched antenna array for physical layer secure communication applications," Progress in Electromagn. Res., vol. 116, pp. 363-379, 2011.

[16] N. Valliappan, A. Lozano, and R. W. Heath, "Antenna subset modulation for secure millimeter-wave wireless communication," IEEE Trans. Commun., vol. 61, no. 8, pp. 3231-3245, Aug. 2013

[17] Q. Zhu, S. Yang, R. Yao, and Z. Nie, "Directional modulation based on 4-D antenna arrays," IEEE Trans. Antennas Propag., vol. 62, no. 2, pp. 621-628, Jan. 2014.

[18] Y. Zhang, Y. Ding, and V. Fusco, "Sidelobe modulation scrambling transmitter using Fourier Rotman lens," IEEE Trans. Antennas Propag., vol. 61, no. 7, pp. 3900-3904, Jul. 2013.

[19] H. Shi and A. Tennant, "Simultaneous, multi-channel, spatially directive data transmission using direct antenna modulation," IEEE Trans. Antennas Propag., vol. 62, no. 1, pp. 403-410, Jan. 2014.

[20] Y. Ding and V. Fusco, "Directional modulation far-field pattern separation synthesis approach," IET Microw., Antennas Propag., vol. 9, no. 1, pp. 41-48, Jan. 2015.

[21] T. Hong, M.-Z. Song, and Y. Liu, "Dual-beam directional modulation technique for physical-layer secure communication," IEEE Antennas Wireless Propag. Lett., vol. 10, pp. 1417-1420, 2011.

[22] T. S. Ng, "Generalised array pattern synthesis using the projection matrix," IEE Proceedings H (IET Microw., Antennas Propag.), vol. 132, no. 1, pp. 44-46, Feb. 1985.

[23] R. Vescovo, "Consistency of constraints on nulls and on dynamic range ratio in pattern synthesis for antenna arrays," IEEE Trans. Antennas Propag., vol. 55, no. 10, pp. 2662-2670, Oct. 2007. 